ÉGYPTE

monde arabe

\section{Égypte/Monde arabe}

$7 \mid 2010$

Fabrique des élections

\title{
Une presse en campagne : la " presse nationale » et l'élection présidentielle égyptienne
}

\section{Enrique Klaus}

\section{(2) OpenEdition \\ Journals}

Édition électronique

URL : https://journals.openedition.org/ema/3004

DOI : 10.4000/ema.3004

ISSN : 2090-7273

\section{Éditeur}

CEDEJ - Centre d'études et de documentation économiques juridiques et sociales

\section{Édition imprimée}

Date de publication : 31 décembre 2010

Pagination : 157-183

ISBN : 978-2-905838-45-2

ISSN : 1110-5097

\section{Référence électronique}

Enrique Klaus, « Une presse en campagne : la « presse nationale » et l'élection présidentielle égyptienne », Égypte/Monde arabe [En ligne], 7 | 2010, mis en ligne le 01 juin 2012, consulté le 07 juillet 2022. URL : http://journals.openedition.org/ema/3004 ; DOI : https://doi.org/10.4000/ema.3004 


\section{RÉSUMÉ}

\section{UNE PRESSE EN CAMPAGNE : LA « PRESSE NATIONALE » ET L'ÉLECTION PRÉSIDENTIELLE ÉGYPTIENNE}

En 2005, I'Égypte a adopté un nouveau mode de désignation de l'exécutif, en remplaçant le référendum à candidature unique sur proposition du Parlement par une élection à candidats multiples au suffrage universel direct. Cet article porte sur la campagne de cette première élection présidentielle, appréhendée à travers le prisme de la " presse nationale » (al-sahâfa al-qawmiyya), c'est-à-dire la presse proche du pouvoir. Celle-ci est prise à la fois comme un objet et un angle d'analyse, pour mieux comprendre les processus liés à la fabrication de cette élection. À travers l'examen détaillé du rubricage des maquettes des trois principales publications et de leur espace publicitaire entièrement dédié à la campagne, l'article s'attache à décrire les modalités pratiques du discours de ce type de presse et, plus particulièrement, les hiérarchisations entre les différentes candidatures et les asymétries que la presse nationale consacre dans sa production, en faveur du président « sortant» Hosni Moubarak. 



\section{UNE PRESSE EN CAMPAGNE : LA « PRESSE NATIONALE » ET L'ÉLEGTION PRÉSIDENTIELLE ÉGYPTIENNE}

e 7 septembre 2005, s'est tenue la " première élection présidentielle de I'histoire égyptienne », comme le rappelaient alors à l'envi les journaux réputés proches du régime. Le scrutin s'est déroulé sur une seule journée, après une campagne électorale officielle de dix-huit jours durant laquelle dix candidats, dont le président sortant Hosni Moubarak, étaient en lice. ${ }^{1}$ Cette élection remplace désormais la candidature unique qui était jusqu'alors proposée par l'Assemblée du peuple puis soumise à référendum. On aurait aimé y voir l'aboutissement des demandes de l'opposition, longtemps répétées, d'abroger le référendum pour lui préférer l'élection², mais ce serait là une vision par trop angélique de la donne politique égyptienne. C'est bien d'un changement " par le haut » dont il s'agit : le président Moubarak est à l'origine de ce changement de procédure, entériné en mai 2005 par l'amendement de I'article 76 de la Constitution, ce qui en limite de facto la portée.

Le point de départ de cet article est la production de la presse dite nationale (al-sahâfa al-qawmiyya) lors de la campagne électorale de l'élection présidentielle. La " presse nationale » désigne ce segment de la presse égyptienne autour duquel s'est constituée l'offre journalistique contemporaine. Née en

1. Il s'agit d'Ayman Nûr (al-Ghad), Nu'mân Gum'a (al-Wafd), Usâma Shaltût (Parti de la solidarité - al-takâful), Wahîd al-Uqsûrî (Parti de l'Égypte arabe et socialiste), Ibrâhîm Turk (Parti unioniste démocratique), Mamdûh al-Qinnâwî (Parti constitutionnaliste, social et libre), Ahmad al-Sabâhî (Parti de la nation), Fawzî Ghazâl (Parti Égypte 2000), Rifa 'at al-'Ajrûdî (Parti de l'entente nationale). La campagne électorale s'est déroulée du 18 août au 4 septembre 2005.

2. À propos des revendications des partis d'opposition participant au «dialogue national » au début des années 1990, cf. Wahîd 'Abd al-Magid, "Crise du dialogue et dialogue de la crise en Égypte. L'expérience du dialogue national de 1994 », Égypte Monde Arabe $\mathrm{n}^{\circ}$ 20, $4^{\mathrm{e}}$ trim. 1994. 
1961, au moment de la nationalisation par Nasser de plusieurs titres de presse et de la fermeture définitive de plusieurs autres ${ }^{3}$, elle coexiste au moment de l'élection avec deux autres types de journaux : les publications liées à un parti politique, réapparues en 1976, sous Sadate, au moment de la réintroduction du multipartisme, et les journaux "indépendants », apparus vers la fin des années 1990 et le début des années 2000, majoritairement financés par des fonds privés, et dont l'indépendance ne peut se comprendre que relativement aux deux autres modèles (national et partisan) présents sur le marché de la presse en Égypte. ${ }^{4}$ La production journalistique en général et celle de la presse nationale en particulier constituent un objet de recherche largement dédaigné par la sociologie politique dans la région. Celle-ci postule la propension de ce segment de la presse à la « langue de bois » et à l'obséquiosité à l'égard des détenteurs du pouvoir, sans jamais interroger les modalités pratiques de son discours. Pourtant, ces journaux constituent l'un des principaux dispositifs d'un régime politique durablement atteint par le "syndrome autoritaire », dont l'un des symptômes est précisément " une tendance au cantonnement de l'expression du pluralisme dans des espaces sous contrôle $» .{ }^{5}$ Comment s'organise en pratique ce cantonnement dans la publicité que suppose la tenue d'une campagne électorale? Quelle est la contribution de ces espaces sous contrôle à l'ingénierie électorale et à la création d'une arène publique instituée et réglementée par la loi électorale ? À partir de ces questions, on montrera dans ce qui suit comment et selon quelles modalités la presse nationale constitue un véritable lieu de fabrication du politique. De fait, la presse nationale est le principal support médiatique des principaux candidats en lice, alors que six d'entre eux ne disposent d'aucun organe porte-voix de leur parti. Bien plus, dans la mesure où ces titres de presse sont parmi les mieux distribués sur le marché égyptien de la presse, ils constituent l'une des principales

3. Cf. Muhammad Hasanayn Haykal, Bayn as-sahâfa wa as-syâssa (Entre la presse et la politique), Le Caire, Dar al-Churûq, 2003, p. 63 et s. ; Sonia Temimi, « Contours d'une identité professionnelle et évolution de son rapport au politique 1923-1970 » dans Égypte Monde Arabe $\mathrm{n}^{\circ}$ 4-5, Le Caire - Bruxelles, CEDEJ / Editions Complexe, 2001, p. 92 et s.

4. Au sujet de la presse dite indépendante, cf. Dina Al-Khawaga, "Sisyphe ou les avatars du nouveau journalisme égyptien » dans Égypte Monde Arabe n 3, 2000, p. $149-175$.

5. Michel Camau et Vincent Geisser, Le syndrome autoritaire. Politique en Tunisie de Bourguiba à Ben Ali, Paris, Presses de Sciences Po, 2003, p. 39. L'étude de von Korff sur la «contribution des journalistes égyptiennes à la démocratisation » serait de nature à relativiser ce dédain. Son intérêt est toutefois limité par les travers téléologiques de l'approche transitologique qu'il adopte : Yorck von Korff, Missing the Wave: Egyptian Journalists' Contribution to Democratization in the 1990's, Hambourg, Deutsches Orient-Institut, 2003. 
sources d'information et de " connaissance pratique ${ }^{6}$ de l'élection pour les lecteurs et les électeurs potentiels. ${ }^{7}$

La présente étude s'appuie sur un corpus de presse constitué sur une période de deux mois (août-septembre 2005), à partir des trois principaux titres de la presse nationale éponymes des groupes de presse qui les éditent : Al-Ahrâm, Al-Jumuhûriyya et Al-Akhbâr (groupe Akhbâr al-Yawm). Cette production journalistique est prise à la fois comme un objet et un angle d'analyse pour mieux comprendre les processus liés à la fabrication de l'élection présidentielle égyptienne. Dans un premier temps, il s'agira de documenter dans le détail comment s'établit en pratique la prédominance du président sortant et comment cette prédominance se fait au détriment des autres candidatures. La presse nationale sera ici étudiée en tant qu'objet dont la production organisée - le maillage des rubriques, les aménagements éditoriaux réalisés pour l'occasion de l'élection - consacre des asymétries et des hiérarchisations entre les différentes candidatures. Dans un second temps, on tentera de sonder le sens que revêt cette élection pour ses participants comme pour divers individus qui, bien qu'en marge de la compétition, ne profitent pas moins du moment privilégié de publicité qu'elle constitue. La presse nationale sera alors considérée comme un angle d'analyse et l'on verra à travers l'examen de messages publicitaires que l'élection permet d'analyser certains mécanismes du fonctionnement du politique en Égypte, notamment les relations entretenues par les hommes d'affaires avec le parti au pouvoir.

\section{PERSPECTIVES ÉLECTORALES ET DÉBATS AUTOUR DE LA PRESSE NATIONALE}

La prépondérance des publications de la presse nationale sur leurs concurrents commerciaux, éditoriaux et politiques est structurellement confortée par le contrôle des principaux groupes de presse " nationaux » (Al-Ahrâm, Akhbâr alYawm, Al-Jumuhûriyya et Dâr al-Hilâl) sur des secteurs d'activité connexes, mais essentiels à la production journalistique, tels que l'impression, la distribution et le secteur des annonces publicitaires. Le marché publicitaire est largement dominé par la filiale Al-Ahram Advertising Company, qui possède 75\% de l'ensemble des concessions à l'échelle du pays, y compris les espaces commerciaux de la presse écrite, I'affichage public dans les aéroports, les stades, les clubs

6. Robert Park, "News as a Form of Knowledge: a Chapter in the Sociology of Knowledge », The American Journal of Sociology, n 45 (5), 1940.

7. Les six candidates dépourvus d'un titre de presse sont les six derniers de la liste établie en note 1 . 
de football, etc. ${ }^{8}$ Cette situation monopolistique permet aux journaux de ces groupes de se tailler la part du lion sur le marché des annonceurs, tout en maintenant leurs concurrents dans une certaine précarité économique.

Dans la perspective de l'élection comme dans son déroulement, les attentes et les actions impliquant la presse nationale sont nombreuses. En amont de la campagne et du scrutin, les enjeux qui entourent son rôle ou son statut dans l'élection dépassent le seul champ médiatique et empiètent largement sur le domaine du politique. Pendant les six mois qui séparent I'annonce de l'amendement constitutionnel (février 2005) du début effectif de la campagne, le statut de la presse nationale est l'objet de vives polémiques dans les milieux journalistiques et politiques. Une campagne de presse initiée en mai par plusieurs journaux de l'opposition conduit en juillet au limogeage des directeurs de publication, dont certains étaient en poste depuis 25 ans, au motif qu'ils avaient dépassé I'âge légal pour assumer leurs fonctions. ${ }^{9}$ En parallèle de ces remaniements, au moment de la discussion au Parlement du projet de loi électorale, les 15-16 juin 2005, les députés du parti au pouvoir, le Parti national démocrate (PND), font front pour que la presse nationale ne tombe pas sous le coup de la loi 174 instituant la neutralité de l'ensemble des médias étatiques (presse audiovisuelle et écrite) et le principe d'égalité de temps de parole entre les candidats. Au moment de ces aménagements législatifs en vue de l'élection, le statut particulier de la presse nationale est largement commenté par la presse d'opposition et apparaît comme l'indice de la faible volonté du régime

8. Source: http://marc.ahram.org.eg/. Les estimations à la même époque de la chambre franco-égyptienne de commerce (L'Antenne, septembre 2005) sont légèrement inférieures à ce chiffre. Selon le site de I'agence, la filiale publicitaire d'AlAhrâm génèrerait $40 \%$ de l'ensemble des revenus du conglomérat. Le groupe Al-Ahrâm contrôle également l'impression de 324 titres égyptiens et étrangers sur deux sites d'imprimerie et la distribution de 7 quotidiens et 26 hebdomadaires, dont un bon nombre de titres de la presse d'opposition partisane et indépendante, en plus des 4 quotidiens et 7 hebdomadaires qu'il édite lui-même.

9. Cette campagne contre les directeurs de presse est inaugurée lors d'un congrès tenu au syndicat, le $1^{\mathrm{er}}$ novembre 2004, par de violentes empoignades entre les quatre membres nasséristes du bureau syndical et des journalistes de la presse nationale. Ces derniers accusaient les premiers « d'offense aux dirigeants journalistiques au sein des institutions nationales (qawmiyya) » (Al-Masrî al-Yawm, 3/11/2004) après que ceux-ci ont proposé d'élire (et non plus de nommer) les directeurs de la presse nationale. Pour plus de détails sur cette affaire, cf. Enrique Klaus, « Le secret de polichinelle : à propos du changement de direction dans la presse d'État » dans Kohstall F. (éd.), L'Égypte dans I'année 2005, Le Caire, CEDEJ, 2006, p. 175-202. 
de tenir des élections libres et concurrentielles. ${ }^{10}$ En somme, qu'il s'agisse de discuter du cadre législatif dans lequel les élections doivent se dérouler, de tenter d'évaluer leur portée à l'aune du rôle de la presse nationale dans la vie publique égyptienne, ou encore d'en profiter pour réformer ce segment de presse, la presse nationale est au centre des débats.

En pleine campagne électorale, les critiques à l'égard de la presse nationale fusent de toutes parts. Un réseau de bloggeurs cairotes relaie ainsi un appel au boycott des différents journaux qui en sont constitutifs pendant toute la durée de la campagne : "Contre la tromperie et la falsification. Pour l'Égypte, ne lisez pas Al-Ahrâm, Al-Akhbâr et Al-Jumuhûriyya » (cf. ci-contre). ${ }^{11}$ Les publications des partis d'opposition ne présentant pas de can-

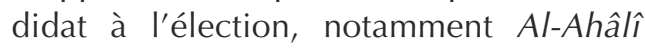
du Tagammu' et Al-'Arabî du Parti nassériste, multiplient elles aussi les critiques à l'égard de la presse nationale et dénoncent

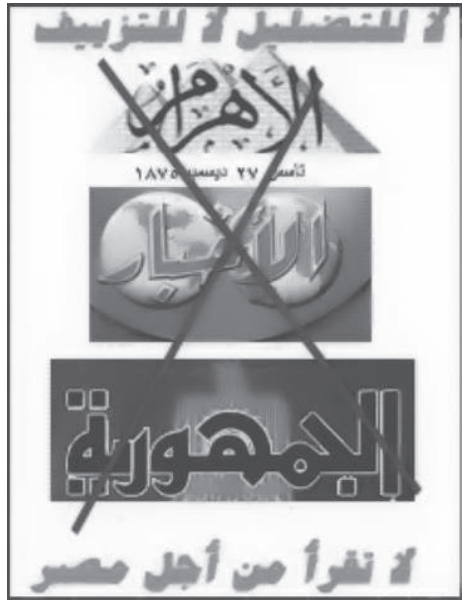

Document 1 la "mascarade du référendum déguisé » (Al-'Arabî, 11/09/2005). De son côté, Mustafâ Bakrî, député indépendant de la gauche islamisée et rédacteur en chef du journal Al-Usbû', choisit le moment de la campagne électorale pour poursuivre, via son hebdomadaire, la campagne de presse lancée plus tôt contre les directeurs de la presse nationale (cf. supra), en publiant une série d'articles contre l'ancien directeur d'Al-Ahrâm et ancien secrétaire du Syndicat des journalistes, Ibrâhîm Nâfi', et en l'accusant de corruption, de détournement de fonds publics et d'enrichissement personnel. ${ }^{12}$

10. Cf. notamment les quotidiens de l'opposition partisane, Al-Ahrâr et Al-Wafd, 17 juin 2005 ; cf. également Ahmad Manîsî, « Al-di'âya al-intikhâbiyya » (la propagande électorale), dans 'Amr Hâchim Rabi'(ed.), Al-ta'dîl al-dustûrî wa-l-intikhâbât al-ri'âssa 2005 (La réforme constitutionnelle et les élections présidentielles de 2005), Le Caire, Al-Ahram Center for Political and Strategic Studies, 2005, p. 322.

11. Cf. http://wa7damasrya.blogspot.com/ (archives : 9/08/2005) et http://misrdigital. blogspot.com/ (archives : 9/08/2005).

12. Cf. Al-Usbû', 22/08/2005. Ibrâhîm Nâfi' a été secrétaire général du Syndicat des journalistes pendant 12 ans (1985-1989, 1993-1996 et 1999-2003). Au moment de cette campagne, C'est un journaliste de l'opposition nassérienne, Galâl 'Ârif (Al-'Arabîl, qui occupe le poste de secrétaire général du Syndicat. 


\section{DU MAILLAGE DES RUBRIQUES AUX POLITIQUES ÉDITORIALES : LA PRÉPONDÉRANCE DE MOUBARAK ET L'EFFACEMENT DE LA CONCURRENCE}

Le rubricage d'une maquette est un outil pratique proposant un dispositif visuel servant à l'élaboration d'une publication, en vue de faciliter sa consommation par les lecteurs. Pour ces derniers, le rubricage est un instrument permettant de guider l'activité de lecture : il leur permet de se repérer dans l'organisation interne du journal et de circuler plus aisément entre les espaces rédactionnels qui en sont constitutifs. Pour les journalistes, la maquette rubriquée d'un journal sert de guide à sa fabrication. En se reportant au « chemin de fer» (expression désignant dans le jargon journalistique l'organisation des rubriques d'un journal), ils maîtrisent les contraintes temporelles inhérentes à sa périodicité, ils évaluent sa complétion, et tentent de respecter les contraintes de format qui s'imposent à la production éditoriale et technique d'une publication régulière. Le rubricage d'un journal est aussi une « machine à solidifier l'événement dans un moule interprétatif ${ }^{13}$ et un dispositif d'ordonnancement de l'actualité consacrant des hiérarchies de discours établies plus ou moins collégialement par la rédaction du journal. L'organisation spatiale et éditoriale des rubriques d'un journal édifie des schèmes de sens de la communication politique autant qu'elle cimente des schèmes de cens de I'expression publique. En d'autres termes, I'organisation rubriquée des discours tenus et/ou rapportés par les journalistes facilite l'expression publique d'un discours légitime sur l'événement et, conséquemment, elle minore un discours «profane " s'y rapportant.

Dès le début du mois d'août 2005, la perspective de la campagne électorale est I'occasion pour Al-Ahrâm, Al-Akhbâr et Al-Jumuhûriyya d'opérer quelques aménagements au sein de leur maquette. Afin de couvrir les activités des participants à la compétition (meetings électoraux, conférences de presse et autres petites phrases), les trois quotidiens créent une rubrique spéciale "Élections », une pleine ou une double-page, annoncée en une et signalée par différents logos aux couleurs du drapeau national. Il s'agit pour ces quotidiens d'agencer au mieux l'espace rédactionnel afin de couvrir l'événement national que constitue l'élection présidentielle, de dégager des pages dédiées à son actualité spécifique, tout en conservant des espaces pour des informations ne s'y rapportant pas directement. ${ }^{14}$

13. Érik Neveu, Sociologie du journalisme, Paris, La Découverte, 2001. p. 49.

14. À titre d'exemple, la mauvaise gestion par l'administration Bush des conséquences du cyclone Katryna en Louisiane, la pression du Conseil de sécurité des Nations-Unies sur la Syrie pour collaborer dans l'enquête sur l'assassinat de Rafîq al-Harîrî, ou encore les diverses rencontres officielles du président Moubarak sont parmi les thèmes qui dominent l'actualité internationale relayée par ces publications. 
Durant la campagne électorale proprement dite, Al-Ahrâm est le plus innovant des trois journaux dans I'aménagement éditorial de sa maquette. ${ }^{15} \mathrm{Du}$ 13 août au $1^{\text {er }}$ septembre 2005, sur une base quasi-quotidienne, la rédaction publie un cahier-supplément de huit pages en quadrichromie, intitulé Al-Risâla (la Lettre). La maquette du supplément se singularise par l'absence totale d'encart publicitaire, à l'avantage de l'espace rédactionnel, comme si toute la superficie du cahier était réservée à l'analyse politique. La maquette est flexible - les articles y sont réunis par la thématique de l'élection - et ne compte que quelques rubriques plus ou moins régulières (cf. infra). En une de chacune des éditions d'Al-Risâla, s'affiche une manchette qui est tour à tour consacrée à des slogans patriotiques ne renvoyant à aucun article spécifique ( "Pour la première fois, le peuple choisit celui qui le gouverne », 13/08/2005 ; "L'Égypte vit une expérience démocratique inédite », 27/08/2005) ou à la promotion de l'intégrité du processus électoral et du scrutin ("Supervision judiciaire complète à l'intérieur et à l'extérieur des bureaux [de vote] ", 22/08/2005; " La supervision des élections est libre et n'est pas soumise au gouvernement »,30/08/2005). La manchette peut également servir à donner ou imprimer un rythme à la campagne ("Dernières heures avant la campagne électorale », 17/08/2005; "Le combat est en marche», 23/08/2005 ; "L'avant-dernière étape dans la course des candidats », 30/08/2005) ou peut renvoyer plus généralement à des enjeux journalistiques typiques du processus électoral, tel que le suivi de campagne ( Nous observons les mouvements des candidats dans les moindres recoins de l'Égypte », 21/08/2005) ou l'analyse politique sur le vif ( $A /$-Ahrâm mène le premier examen entre les candidats à la présidentielle », 25/08/2005). La page de une de ce cahiersupplément est consacrée à l'information et réunit des brèves sur l'actualité des divers candidats, annonçant généralement leurs déplacements, mettant en lumière le rôle innovant $d^{\prime}$ Internet ${ }^{16}$, ou encore, entretenant le consensus contre "l'ingérence étrangère » que constituerait l'observation du scrutin par des organismes internationaux. Les articles de pages centrales sont majoritairement orientés vers l'analyse journalistique et s'attachent à traiter différents aspects du processus électoral à travers des thématiques diverses : les formes

15. À l'exception du magazine hebdomadaire Rûz al-Yûsif, hors l'échantillon de cette étude, qui s'est lancé à l'assaut du marché de la presse quotidienne à la veille de la campagne électorale, le 15/08/2005. Son rédacteur en chef n'est autre que 'Abdallâh Kamâl, membre du Comité des politiques du PND, dirigé par Gamal Moubarak. Notons également, du côté de la presse d'opposition, que I'hebdomadaire éponyme du parti al-Ghad est paru quotidiennement à partir du 17/08/2005 et ce, jusqu'à peu après la campagne électorale.

16. Sur les sites de Moubarak, Gum'a et Nûr, cf. Al-Risâla, 21, 23, 30/08/2005 ; sur le site "Shâyfin-kum »(On vous voit), cf. Al-Risâla, 28/08/2005. 
de la propagande électorale, le vote féminin ou la question de la réforme constitutionnelle. ${ }^{17}$ Outre ces articles de facture classique que I'on retrouve à l'intérieur d'Al-Risâla, la rédaction entretient régulièrement trois rubriques ( "La rue politique », «Confrontation » et «Témoignages de proximité ») dont la gestion éditoriale reflète les orientations politiques de l'équipe de rédaction.

Sur la page "La rue politique », un encadré est réservé à I'interview des candidats en lice, donnant ainsi à voir le pluralisme partisan de rigueur en cette période électorale. Chacun des candidats est ainsi dûment interviewé, de manière conventionnelle, à propos de ses activités, de son programme ou, à défaut, de son " message à la nation ». Pour autant, autour de ce seul et même exercice journalistique, des différences de traitement entre les candidats apparaissent de manière claire. Comme toutes les interviews présidentielles, celle du « candidat du PND» fait la une et remplit les premières pages d'Al-Ahrâm $(2 / 09 / 2005)$ et ce, quelques jours avant la tenue du scrutin. Le candidat du Néo-Wafd, Nu'mân Gum'a, n'a pas droit aux mêmes égards, mais il inaugure tout de même la rubrique d'Al-Risâla peu avant l'ouverture de la campagne $(14 / 08 / 2005)$ et se voit gratifié plus tard d'une deuxième interview dans la rubrique «Élections » d'Al-Ahrâm (29/08/2005), le donnant ainsi à voir comme le concurrent principal du président sortant. Les huit autres candidats, ne bénéficient que $d^{\prime}$ une seule interview et leurs propos sont systématiquement renvoyés dans le cahier-annexe d'Al-Ahrâm.

Cette hiérarchisation se vérifie dans une autre rubrique d'interviews placée sur la même page et donnant la parole aux épouses des candidats. Les conjointes des candidats y sont interrogées, à l'exception notable de Madame Gum'a et de la première dame d'Égypte dont les activités caritatives font I'objet de longs articles dans $A$ l-Ahrâm. ${ }^{18}$ On peut en déduire que cette rubrique vise à pallier le déficit de notoriété des candidats anonymes (cf. infra), par le truchement d'une forme de "peopolisation » de la politique inédite dans l'histoire du plus vieux titre égyptien. Cependant, I'adoption de cet angle journalistique spécifique participe de la marginalisation de ces candidats au moment même où un espace rédactionnel leur est consacré. Celles qui partagent la vie des candidats inconnus étant elles-mêmes de parfaites inconnues ${ }^{19}$, cet angle désamorce d'emblée l'espace médiatique qui leur est offert de toute charge politique et de toute possibilité de capitalisation électorale.

Ainsi observe-t-on à travers l'exercice de l'entretien journalistique la construction d'une grille de lecture éditoriale de la compétition électorale qui, bien que présentée comme pluraliste, se révèle favorable au président

17. Cf. respectivement Al-Risâla, 25/08/2005, 27/08/2005 et 28/08/2005.

18. Cf. Al-Ahrâm, 10, 14, 16, 27, 28/08/2005 et 1, 2/09/2005 (liste non-exhaustive). 19. À l'exception toutefois de l'épouse d'Ayman Nûr ; jusqu'à la candidature de son mari, Gamîla Ismâ^îl était animatrice de télévision. 
sortant. Lors d'élections législatives précédentes, on a pu observer de la même manière comment la presse nationale pouvait être un instrument de qualification ou de disqualification des prétendants à I'activité politique. La presse nationale avait déjà été le lieu de ce jeu politique en se faisant le chantre de l'unité nationale avant le début de la campagne, dans le but d'attirer les partis d'opposition sur le terrain électoral pour ensuite, sitôt la compétition électorale lancée, mener de virulentes campagnes de dépréciation contre les candidats de ces mêmes partis. ${ }^{20}$ Pareille entreprise dépréciative est observable en 2005 dans les colonnes d'Al-Risâla, comme le montre notamment I'interview d'Ayman Nûr, la seule, publiée en début de campagne dans la rubrique «La rue politique»:

«Bien qu'il se soit opposé aux garanties qu'englobait l'amendement de l'article 76 de la Constitution, il a posé sa candidature au poste de président de la République. Bien qu'il ait [d'abord] refusé le soutien financier proposé par l'État, pour que chaque candidat puisse mener sa campagne électorale, il s'est rétracté et a fini par accepter le soutien de l'État... Alors qu'est ce qui a poussé le Dr Ayman Nûr, président du parti al-Ghad, à poser sa candidature pour le poste présidentiel malgré son opposition aux garanties de I'article 76, et à accepter le soutien [financier] de l'État alors qu'il l'avait [initialement] refusé ? Des questions brûlantes et des réponses qui font monter la température dans le dialogue suivant, en toute sincérité (...) ». ${ }^{21}$

Conventionnellement, une interview est introduite par un court paragraphe, le «lead», qui vise à livrer au lecteur quelques éléments contextuels avant de livrer la teneur de l'entrevue entre le journaliste et la personnalité interrogée. ${ }^{22} C^{\prime}$ est bien le cas dans le lead de l'interview d'Ayman Nûr, mais le choix de ces éléments de contexte est clairement dépréciatif, dès lors que la rédaction le décrit en quelques lignes comme une personne inconstante, indécise, réfractaire au changement, vénale et inconséquente. Mais le travail éditorial entourant la publication de cet interview ne s'arrête pas là. Le titre choisi par la rédaction ne concerne en rien ses activités de campagne, et se lit

20. Dina Al-Khawaga, «Le Parti national démocrate et les élections de 1995 : conjonction de nombreuses logiques d'action » dans Gamblin S. (dir.), Contours et détours du politique: les élections législatives de 1995, Paris, L'Harmattan/CEDEJ, 1997, p. 90-91.

21. Al-Risâla, 21/08/2005. Dans une interview publiée par la rédaction de Al-Ahram Weekly (18/08/2005), Ayman Nûr souligne que son parti n'avait pas refusé I'aide financière de l'État dans le cadre de sa campagne, mais qu'il avait refusé l'aide octroyée aux partis politiques.

22. Cf. Steven Clayman et John Heritage, The News Interview. Journalists and Public Figures on the Air, Cambridge, Cambridge University Press, 2002. 
comme une citation d'Ayman Nûr : "Les Frères [musulmans] ont le droit de fonder un parti civil ». Le thème de l'islam politique est motivé par la récente visite d'Ayman Nûr au Guide suprême Mahdî 'Âkif. Il est à noter cependant que ni la rencontre entre Mahdî 'Âkif et Nu'mân Gum'a, le 14 août, ni celle, le lendemain, du même 'Âkif avec un autre candidat, Fawzî al-Ghazâl, n'ont été mentionnées dans leurs entretiens respectifs tels que ceux-ci ont été publiés par Al-Risâla. II s'agit pour le titreur d'Al-Risâla de souligner la tolérance du candidat $d^{\prime}$ al-Ghad à l'égard $d^{\prime}$ un groupe politique «interdit » - parce que partisan d'une ligne religieuse rigoriste voire littéraliste - mais « toléré » - par nécessité de survie d'un régime en perpétuelle recherche de légitimité. Les relations de Nûr avec la confrérie font ainsi l'objet de trois des sept questions qui lui sont posées dans cette interview aux allures d'interrogatoire ; les quatre autres, non moins "suspicieuses", concernent les conditions matérielles et financières de sa campagne. À l'opposé, en regard direct de l'interview de Nûr, un entretien avec le Shaykh d'al-Azhar, Muhammad al-Tantâwî, est publié hors rubricage, bien qu'il reprenne la mise en page de la rubrique "Témoignage de proximité », exclusivement réservée au bilan élogieux du règne de Moubarak (cf. infra). Tantâwî se réfère exclusivement à ce dernier, dont il souligne le rôle de protecteur d'un islam sunnite, quiétiste et « docile», dans des propos également repris en titre: « II est le président qui a le plus protégé le rôle d'al-Azhar, en Égypte et à l'étranger ». La comparaison entre les deux interviews est fortement suggérée par la mise en page de la maquette et par leur mise en regard. Le thème commun de la religion fonde la comparaison et permet de disqualifier la conduite d'Ayman Nûr pour mieux mettre en avant celle de Hosni Moubarak.

L'angle comparatif est également consacré dans la maquette d'Al-Risâla, dans une autre rubrique intitulée "Confrontation»(muwajaha). La comparaison qu'induit l'intitulé de la rubrique est certes postulée mais ne se vérifie pas à la lecture des articles qui s'y succèdent. II ne s'agit pas de confronter les candidats entre eux mais, selon le texte introductif, de " confronter un problème ou une crise dont nous souffrons [aux idées] d'un homme qui en a la solution ». Au fil des éditions, cette rubrique irrégulière des pages centrales s'est révélée très malléable et réunit autant d'interviews que d'articles d'opinion. Qu'il s'agisse d'universitaires ou de membres du gouvernement, tous les participants à cette rubrique sont unis par un même soutien au " président Moubarak ». Ainsi, dans son édition du 31 août, la "confrontation » se résume à une mise en regard des analyses d'Ahmad Abû-I-Ghayt, ministre des Affaires étrangères, et de celles de Fârûq Husnî, ministre de la Culture. Le choix de ces deux ministres ne doit rien au hasard. Les portefeuilles ministériels des deux hommes leur permettent d'aborder les thèmes de prédilection de la campagne de Moubarak : la politique étrangère, point fort du président, et la liberté d'expression, métonymique des libertés publiques. Moubarak y est présenté d'un côté sous les traits d'un homme de paix et de médiation et, de l'autre, en défenseur des droits de l'homme. 
Le soutien apporté à Moubarak prend diverses formes au sein de ces aménagements éditoriaux et il est même intégré à la maquette, dans une rubrique plus ou moins régulière $\mathrm{d}^{\prime} A /$-Risâla, entièrement consacrée au président Moubarak. ${ }^{23}$ La rubrique "Témoignages de proximité " (shahâdât 'an qurb) est placée au centre de la maquette, sur une pleine page, et propose au lecteur un long article laudateur, signé par des éditorialistes ou des universitaires habitués des pages opinions d'Al-Ahrâm. L'angle éditorial est parfois précisé en introduction de l'article comme suit :

«Ces témoignages sont ceux d'un passé récent, qui dessine les contours de l'avenir de l'Égypte. Ce sont des témoignages d'hommes qui ont approché le président Moubarak, qui ont vécu avec lui les moments de rupture et l'ont trouvé robuste, convaincu que la victoire vient du cœur de la défaite; ils ont vécu avec lui les victoires et ils l'ont trouvé modeste, épargné par les tentations de l'euphorie victorieuse et déjà à la recherche d'un nouveau rêve pour cette nation [...]. Ces témoignages présentent des détails, des histoires et des données qui, dans leur ensemble, modèlent l'image du président, du dirigeant et de I'homme ».

Dans ce texte qui tient lieu de synopsis à la série d'articles, il n'est pas explicitement question de l'élection présidentielle, ni de la candidature de Moubarak. L'insertion de cette pleine-page dans le supplément du journal suffit cependant à la replacer dans le contexte de la compétition électorale. Le texte place d'emblée cette production journalistique dans une organisation séquentielle du temps, opposant passé et futur, qui relie le bilan du règne de Moubarak à la perspective de sa réélection. La rubrique réunit des textes ayant valeur testimoniale, écrits par des personnalités censées faire partie de l'entourage présidentiel. La tonalité laudative de ces textes est annoncée dès l'introduction qui projette une image idéalisée de la fonction présidentielle (force de caractère, modestie, ambition) au service de la nation. Le texte introductif de la rubrique place l'article du jour dans un feuilleton journalistique et la compilation de ces "témoignages », " détails, histoires et données » confondus, confère un statut documentaire au texte publié, lequel serait en mesure de donner un reflet fidèle des multiples facettes de Moubarak, «le président, le dirigeant et l'homme».

La rubrique est lancée peu avant le début de la campagne, dans les pages d'Al-Ahrâm, vraisemblablement dans le but d'attirer les lecteurs vers cet espace éditorial. Elle est inaugurée par le conseiller politique du président, Usâma al-Bâz, qui vise très explicitement dans son intervention l'élection

23. À la notable exception d'une édition de début de campagne consacrée à Nu'mân Gum'a : "Nu'mân Gum'a comme je le connais » par le militant wafdiste Sulaymân Gûda, Al-Risâla, 22/08/2005. 
présidentielle : "Le maintien de Moubarak pour cimenter la sécurité du pouvoir est une garantie de stabilité pour la nation et ses fils " (Al-Ahrâm, 13/08/2005). Puis c'est au tour des éditorialistes de faire l'éloge du président Moubarak. Sur un ton obséquieux, Anîs Mansûr lance en titre : "Que Dieu te garde, président ! Comment ne pas choisir Moubarak comme président pour l'Égypte »(Al-Ahrâm, 14/08/2005). Ibrâhîm Nâfi' prend ensuite le relais, le premier jour de la campagne électorale, et ouvre son article en revenant sur les souvenirs de leurs premières rencontres, alors que Moubarak n'était encore que le vice-président d'al-Sadate. II égrène ensuite les " exploits » (injâzât) du président Moubarak, de la paix régionale à la construction du réseau routier national en passant par l'introduction des téléphones fixes puis cellulaires en Égypte. À nouveau, dans le "témoignage » de Nâfi', la perspective de l'élection reste au premier plan et le ton patriotique et solennel est de circonstance : "Ô Égypte, Moubarak se tient maintenant au seuil de l'étape d'une grande effervescence » (Al-Ahrâm, 18/08/2005). Des personnalités publiques prennent également la plume, tel Zâhî Hawwâs, le très médiatique directeur du Conseil suprême des antiquités ${ }^{24}$, qui martèle que "La civilisation égyptienne est son objectif premier » (Al-Risâla, 24/08/2005), ou encore le pape Shenouda III, pour I'occasion chantre de I'unité nationale (Al-Risâla, 14/08/2005).

À travers I'analyse du maillage des rubriques d'Al-Risâla, on s'aperçoit que le président Moubarak occupe un espace démesuré au regard de la place accordée aux neuf autres candidats et que sa promotion se fait sur l'ensemble des rubriques des journaux. Les rubriques classiques de l'expression politique (actualité politique, opinion) sont autant employées à promouvoir le nouveau mode de scrutin qu'à soutenir la candidature Moubarak, I'un appelant l'autre. Les pages "Opinions », tout particulièrement, rassemblent plusieurs contributions appelant les citoyens à la participation ${ }^{25}$, omettant rarement de lier la tenue de ces élections à l'initiative de Moubarak d'amender la Constitution. Mais ce n'est pas tant dans ces rubriques traditionnelles de l'expression politique que la prédominance du discours pro-Moubarak est la plus éloquente. Au-delà de l'élection, ces rubriques poursuivent une politique rédactionnelle

24. À propos des prestations médiatiques de Hawwâs, cf. Jean-Gabriel Leturc, «Et Zahî créa l'Égypte : quand glamour et patrimoine défraient la chronique » dans Klaus E. et Hassabo C. (dirs.), Chroniques égyptiennes 2006, Le Caire, CEDEJ, 2007.

25. Entre autres exemples : le dossier spécial d'Al-Jumuhûriyya « Nous sommes tous Égyptiens: les élections sont une occasion de rapprochement entre le gouvernement et le peuple » (4/08/2005); les dossiers d'Al-Ahrâm, "Appel à la participation » (6/09/2005), «Participez, ne boycottez pas » (7/09/2005) et son article « Les oulémas : la participation est un devoir légitime (char $\hat{\imath}) »($ id.). 
habituelle de promotion des activités présidentielles. ${ }^{26}$ II est sans doute plus significatif de relever la contribution de rubriques « ludiques » d'information ou magazine à la promotion de la candidature de Moubarak. La rubrique d'actualité féminine, entre autres exemples, relaie sous la forme d'une consultation populaire des doléances personnelles au côté de déclarations de soutien ou d'intention de vote en faveur de Moubarak, sous le titre "les réclamations adressées au Président par la femme modeste de la rue égyptienne pour le prochain mandat présidentiel » (Al-Ahrâm, 2/09/2005).

La prépondérance éditoriale et politique du candidat Moubarak est d'autant plus manifeste que, dans leurs déclarations relayées par la presse nationale, les candidats des petits partis font montre d'un rapport particulier à la compétition en neutralisant d'emblée la concurrence que leur présence est supposée introduire. Dès le début de la campagne, Al-Ahrâm rapporte les grandes lignes d'un discours d'Usâma Shaltût sous un titre accrocheur : "Ce serait un sacrilège si quelqu'un d'autre que moi gouvernait l'Égypte (harâm an yahkum misr ahad ghayrî) » (Al-Ahrâm, 21/09/2005). Si la déclaration semble péremptoire dans l'intitulé, les propos du candidat, non-tronqués dans l'article, montrent que Shaltût s'oppose en réalité à huit adversaires, et non à neuf, excluant la candidature de Moubarak de la compétition. Le refus de se positionner en concurrent du président peut prendre des formes inattendues comme en témoignent les propos d'Ahmad al-Sabâhî qui se démarque des autres candidats par son soutien inconditionnel à Moubarak : «Élisez Moubarak, car il est le plus apte à diriger le pays à cette étape-ci, et si vous ne lui donnez pas votre voix, ne m'en honorez pas » (Al-Ahrâm, 2/09/2005). Le candidat appelle ainsi les électeurs au "vote utile ", si l'on peut dire, et en arrive à faire campagne pour une candidature adverse à la sienne. L'état de l'offre électorale explique en partie ce rapport pour le moins particulier à la concurrence dans le cadre d'une compétition électorale. Le fait est que la majorité des candidats est inconnue du grand-public : sur dix candidatures, on en relève sept - ou huit selon que l'on inclut ou pas Ayman Nûr - sans passé politique de notoriété publique. Comme le souligne Wahîd al-Uqsûrî peu avant l'ouverture de la campagne: "L'homme de la rue ne ressent pas la présence des partis d'opposition et peut-être ne connait-il même pas le nom de la plupart d'entre

26. C'est le sens d'une critique de l'hebdomadaire satirique d'opposition, Al-Dustûr : "Les rédacteurs en chef de notre presse nationale (qawmiyya) s'imaginent que, quand ils utilisent [l'expression] de 'citoyen Hosni Moubarak' ou de 'candidat du PND' à la place de 'président Moubarak', ils se moquent du lecteur. Ils vivent dans I'illusion qu'eux et leurs journaux sont neutres et [équilibrés] entre le président et la concurrence. Mais ce jeu douteux ne berne personne. Le candidat Hosni Moubarak ou le citoyen, appelez-le comme vous voulez, n'a cessé d'occuper la une et les manchettes de ces journaux tout au long des années passées sous sa présidence » (Al-Dustûr, 24/08/2005). 
eux »(Al-Jumuhûriyya, 3/08/2005). Ce phénomène, qui n'est pas spécifique à ce scrutin présidentiel ${ }^{27}$, et qui souligne le problème structurel du renouvellement du personnel politique en Égypte, n'est pas sans conséquence sur la candidature de ces anonymes.

L'anonymat d'une majorité de candidats prenant part à la compétition a deux implications principales dans leur performance électorale et, au-delà, dans l'élection en tant que telle. La première a trait aux modalités de la propagande électorale de ces candidats anonymes, chefs de file de petits partis politiques. II n'est pas rare que ceux-ci profitent des interviews journalistiques pour se faire connaître, non sans quelque maladresse, en rendant public leur curriculum vitæ. Sur le seul encart publicitaire qu'il publie en une d'Al-Ahrâm (3/09/2005), Usâma Shaltût met ainsi en avant son parcours universitaire, ses expériences militaires, tant théoriques que pratiques, et son expérience politique au sein des institutions de l'État :

«Il plut à Dieu de m'offrir nombre de qualifications [...] pour me permettre de prendre part à la compétition. Parmi les plus importantes : j'ai étudié la comptabilité et l'économie, ainsi que les études africaines et les sciences stratégiques dans le domaine des missiles, j'ai participé à la guerre d'octobre [1973] et j'ai joué un rôle important en haltérophilie. Puis, je suis entré en politique en devenant membre de l'Assemblée consultative (al-shûrâ), en parallèle de ma carrière de professeur de commerce à l'université de Béni Souef $» .^{28}$

La seconde implication de l'anonymat des candidats réside dans la poursuite d'objectifs non-électoraux par une majorité des candidatures en lice. Le corollaire de la non-concurrence au président est que les candidats anonymes ne visent pas le résultat du scrutin, mais prennent part à l'élection en cherchant à capitaliser en termes d'écologie partisane, pour faire connaître leur parti et tenter ainsi d'augmenter le nombre de leurs militants. Ainsi, quelques jours avant le scrutin, Usâma Shaltût soutient que " [son] but dans le combat électoral est de faire connaître [son] parti auprès des masses » (Al-Ahrâm, 2/09/2005). Interrogé sur son anonymat lors d'une conférence de

27. Assia Boutaleb, "The Parliamentary Elections of Year 2000 in Egypt: a Lesson in Political Participation », Cairo Papers in Social Science, 25 (1-2), Cairo, AUC Press, 2004, p. 20.

28. Al-Akhbâr, 18/08/2005. Dans les courriers de lecteur sélectionnés par la rédaction, un colonel à la retraite se plaint lui aussi de l'anonymat de la plupart des candidatures et propose, pour y remédier d'allonger la période de la campagne électorale et de " publier la liste des 21 partis politiques égyptiens, la carte d'identité des candidats ainsi que leurs CV, depuis leurs dates de naissance jusqu'au 6 septembre 2005, ainsi que leurs programmes politiques que nous conserverions en tant que documents de référence pour l'avenir » (courrier des lecteurs, Al-Ahrâm, 18/08/2005). 
presse préélectorale, Wahîd al-Uqsûrî déclare avoir deux objectifs en prenant part à la « compétition » électorale :

«Parvenir au poste de président, malgré les difficultés du combat [...], et diffuser le programme et les principes du parti et, ce faisant, rassembler le plus grand nombre de citoyens afin d'investir cela dans le renforcement des bases du parti, avec l'entrée de nouveaux cadres dans ses rangs $»{ }^{29}$

Pour ces chefs de partis marginaux, la campagne électorale est l'occasion de capitaliser en termes d'effectifs partisans et non de s'efforcer de réduire l'étroitesse de leur clientèle électorale. En dépit de leur participation, ces candidats visent des enjeux qui ne sont pas directement liés à la compétition électorale, si ce n'est sur le long terme, dans la perspective de scrutins ultérieurs. L'intérêt pour l'élection se déplace ainsi de l'issue du vote vers la campagne électorale, catalyseur de la visibilité publique d'individus qui ne prétendent pas à l'investiture suprême, mais seulement à la notabilité politique. Ceci n'est pas dénué de conséquences sur la compétition électorale, dès lors désamorcée de tout enjeu de pouvoir. C'est bien cela qui caractérise le mieux l'absence d'enjeu d'alternance dans cette élection dont la presse nationale, à sa manière, rend parfaitement compte.

\section{LA CAMPAGNE DANS L'ESPACE PUBLICITAIRE DES JOURNAUX}

L'analyse de la partie rédactionnelle des journaux de la presse nationale a permis de documenter l'asymétrie qui règne entre les candidatures et la domination du président Moubarak dans la couverture de la campagne électorale par ces journaux. Cette « asymétrie éditoriale » prend diverses proportions selon les trois publications de notre échantillon. Au moment du scrutin, trois études d'ONG publiées dans Al-Ahrâm (7/09/2005) relèvent que Al-Jumuhûriyya consacre $65 \%$ de la surface du journal à l'actualité du candidat Moubarak, et $9 \%$ à ses activités présidentielles, ne laissant ainsi que $26 \%$ aux neuf autres candidats. Al-Ahrâm réserve quant à lui $57 \%$ de son espace éditorial à la couverture des activités du candidat Moubarak et $12 \%$ à ses activités présidentielles. Sur l'ensemble des publications de la presse nationale, la couverture médiatique de Moubarak est estimée par la Commission de suivi des médias du Conseil national de supervision des élections à 59\% de la superficie éditoriale totale, les neuf autres candidats se partageant le reste. ${ }^{30}$ Bien qu'édifiants, ces chiffres sont en-deçà de la réalité de ce qu'il est donné à voir au lecteur, dès lors qu'ils ne comptabilisent pas l'espace publicitaire.

29. Al-Jumuhûriyya, 3/08/2005.

30. Al-Misriyyûn, 25/08/2005. www.almesryoun.com/ShowDetails. asp? NewID=3677\&Page $=1$ 
Or, I'examen de ce secteur essentiel à l'économie et à la survie d'un journal permet de rendre d'autant plus saillante l'asymétrie flagrante qui sépare les candidats et la prépondérance manifeste de Moubarak dans la compétition électorale et les modalités particulières qu'elle emprunte. Que ce soit par choix politique et/ou par nécessité budgétaire, la majorité des candidats ne diffuse pas de publicités dans la presse nationale. À l'exception d'un encart de petit format d'Usâma Shaltût paru en une d'Al-Ahrâm (3/09/2005 ; cf. supra), le candidat du PND et celui du Wafd se partagent seuls (ou presque) l'espace publicitaire des journaux de la presse nationale. Nu'mân Gum'a est le candidat qui diffuse le plus de publicités électorales dans ces journaux et, à intervalles réguliers pendant la campagne, son quartier général de campagne loue des pleines pages à l'intérieur de la maquette pour publier les affiches officielles de sa campagne axée sur le changement ( $Y$ en a marre ! - Avec moi, ô peuple, changeons l'Égypte pour de vrai »; "La garantie, le peuple a choisi le changement et des juges libres et dignes »). Quant au PND, il ne publie que deux affiches officielles en début et fin de campagne (Al-Ahrâm, $17 / 08$ et 04/09/2005), en quatrième de couverture, c'est-à-dire la concession publicitaire la plus visible et, de ce fait, la plus onéreuse. Malgré ce faible nombre de publicités officielles, le déséquilibre visible dans les espaces publicitaires se fait à l'avantage du candidat PND.

L'essentiel de la campagne publicitaire de soutien à Moubarak est le fait d'individus et notamment d'entrepreneurs du secteur privé. Qu'il s'agisse de directeurs de PME locales ou de PDG de grands conglomérats, ces annonceurs d'un type particulier cumulent le double avantage d'une opération marketing pour leurs entreprises et de la publicité de leur soutien à Moubarak. S'ils ne participent pas directement à l'élection, ils visent toutefois, par voie de presse et par le biais de l'annonce publicitaire, des objectifs semblables à ceux des candidats anonymes, en investissant dans l'élection et en investissant la campagne électorale.

Les principales zones d'achalandage des journaux se distribuent entre la une (encart 20x8cm), la quatrième de couverture (pleine ou demie-page) et les pages d'actualités sportives (divers formats). On y trouve habituellement des annonces publicitaires pour des produits commerciaux et des offres de service du secteur privé comme du secteur public. On peut également y lire des messages personnels dont l'objectif n'est pas commercial : il s'agit d' « appels à l'aide » (istigâtha), qu'un individu ou un groupe de personnes peut publier à ses frais pour réclamer un arbitrage ou une intervention dans une affaire personnelle et invoquer les plus hauts échelons de l'État, prioritairement le président de la République et les membres du gouvernement. En période électorale, l'appel à l'aide est concurrencé par d'autres formes de messages personnels à visée essentiellement laudative. Les quelques appels à l'aide publiés lors de la campagne sont toutefois fortement influencés par le contexte électoral. Dans I'édition d'Al-Ahrâm du 26/8/2005, les employés d'une entreprise du secteur pétrolier publient un appel à l'aide qui n'est, dans un premier 
temps, pas adressé au président Moubarak, mais au Premier ministre et au ministre du Pétrole. Ils exposent plusieurs revendications d'ordre corporatiste (primes annuelles impayées, augmentation de salaire, prime au logement), sans faire aucune référence au contexte électoral. Le 6 septembre, les mêmes employés publient un deuxième encart reprenant l'affiche de campagne de Moubarak, avec une argumentation beaucoup plus en phase avec la majorité des annonces personnelles publiées en cette période électorale :

"Oui à Moubarak, du plus profond des cœurs, pleins d'amour et de gratitude pour ce qu'il présente à l'Égypte chérie

L'ensemble des ouvriers du centre médical pour le pétrole, basé au Caire, soutiennent Monsieur le président Mohamed Hosni Moubarak et présentent leurs remerciements et leur estime à :

Son Excellence le Premier ministre

Son Excellence Monsieur I'Ingénieur ministre du Pétrole [...]

Pour avoir répondu favorablement à l'appel à l'aide publié dans le journal Al-Ahrâm du vendredi 26/8/2005 et pour [leur] avoir restitué[s] l'ensemble de leurs droits, au même niveau que l'ensemble de leurs collègues du secteur pétrolier, dans le climat démocratique et sous la guidance de son Excellence le président

[Signé :] L'ensemble des ouvriers du centre médical pour le pétrole, Le Caire » (Al-Ahrâm, 6/09/2005)

Si la demande n'était pas adressée, dans un premier temps, au président Moubarak, on y évoquait déjà « le climat démocratique et [...] la guidance de son Excellence le président ». Après avoir été entendus, vraisemblablement, les employés de l'entreprise pétrolière ont choisi de politiser l'enjeu du succès de leurs revendications et de le rendre public afin de participer à leur manière à la campagne électorale de Moubarak. Peu avant cette dernière annonce, un autre appel à l'aide publié par les ayant-droits de la caisse de retraite de la Misr International Bank, est quant à lui directement indexé sur la période électorale et mêle une forme de soutien électoral à la requête :

"Allégeance (mubâyy'a) et appel (nidâ')

Les familles de 1600 employés et notables des retraites de la Banque internationale Misr (MIB) [prêtent] allégeance à Monsieur le président M. H. Moubarak et soutiennent son programme électoral.

Et ils conjurent (yunâshidûn) le Premier ministre et les ministres en poste (ministre de I'Investissement, ministre des Assurances et des Affaires sociales, ministre de l'Économie, le gouverneur de la Banque centrale) de suivre la méthode du président de la République et les fermes assurances que contient son programme électoral pour activer des caisses d'assurance privées et alternatives.

Ils [les] prient d'intervenir pour arrêter ou prévenir toutes les décisions et propositions actuellement discutées à la Banque centrale 
avec la banque Misr et le conseiller juridique de la banque acheteuse, pour publier des décisions de la Banque centrale ou des propositions $\mathrm{d}^{\prime}$ amendement judiciaire. Tout ceci dans le but de liquider les caisses d'assurance alternatives des employés de banque, ce qui est en contradiction avec les orientations de Monsieur le président de la République et de son programme électoral. » (Al-Ahrâm, 4/09/2005)

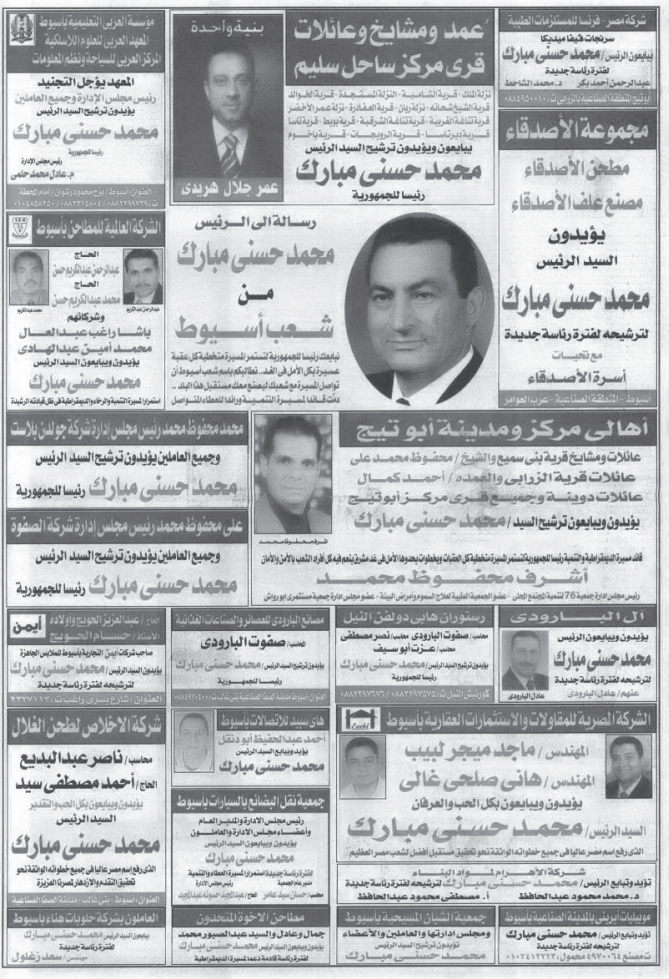

Document 2 : (Al-Akhbâr, 28/08/2005)
On voit dans cette annonce combien la campagne électorale constitue un moment privilégié pour présenter des doléances et des requêtes particulières. Dans cette entreprise, le programme électoral du président fait l'objet de nombreux investissements : on lui prête soutien comme on soutient un candidat, on lui prête la valeur d'une feuille de route s'imposant au gouvernement et I'on s'en saisit en dernier recours pour faire pression sur les décideurs visés par l'annonce. On observe là une figure particulière de I'affaire, au sens d'une "forme politique critique ${ }^{31}$ dont on voit poindre à travers l'adresse au président et le

31. Sur ce point, cf. Luc Boltanski, L'amour et la justice comme compétences. Trois essais de sociologie de l'action, Paris, Métailié, 1990 ; Élizabeth Claverie, "Procès, affaire, grandes causes: Voltaire et l'innovation critique», Politix, vol. 7, $\mathrm{n}^{\circ} 26$, 1994, p. 76-85 ; Élizabeth Claverie "La naissance d'une forme politique : I'affaire du chevalier de La Barre » dans Roussin P. (dir.), Critique et affaires de blasphème à l'époque des Lumières, Paris, Honoré Champion, 1998, p. 185-260 ; Luc Boltanski et Élizabeth Claverie, "Du monde social en tant que scène d'un procès » dans Boltanski L., Claverie É., Offenstadt N., van Damme S. (dirs.), Affaires, scandales et grandes causes : de Socrate à Pinochet, Paris, Stock, 2007, p. 395-452. 
respect des hiérarchies de l'exécutif, I'une des caractéristiques essentielles, à savoir « le lien étroit qu'elle entretient à la légitimité et aux dispositifs de pouvoir se réclamant $d^{\prime}$ une légitimité ${ }^{32}{ }^{32}$

La campagne électorale, en tant qu'événement public, se présente pour ces annonceurs comme le catalyseur efficace d'une annonce publique devant permettre d'obtenir gain de cause dans des litiges particuliers. Outre ces messages de doléance, des messages personnels de soutien à Moubarak paraissent dans la presse nationale lors de la campagne électorale. Pour les accueillir et les publier, les rédactions de la presse nationale aménagent de nouveaux espaces de publicité, en intercalant des feuillets non-rédactionnels au sein de la maquette de plus en plus régulièrement à l'approche du scrutin. Sur ces pages, le service commercial des publications regroupe les petits encarts d'annonces en fonction de la région d'origine de l'annonceur, généralement patron d'une PME. La page ci-contre (Document 2) réunit les annonces de ces petits patrons et/ou notabilités locales de la région d'Assiout. On retrouve ce type d'annonce dans les espaces commerciaux habituels de la maquette, aux

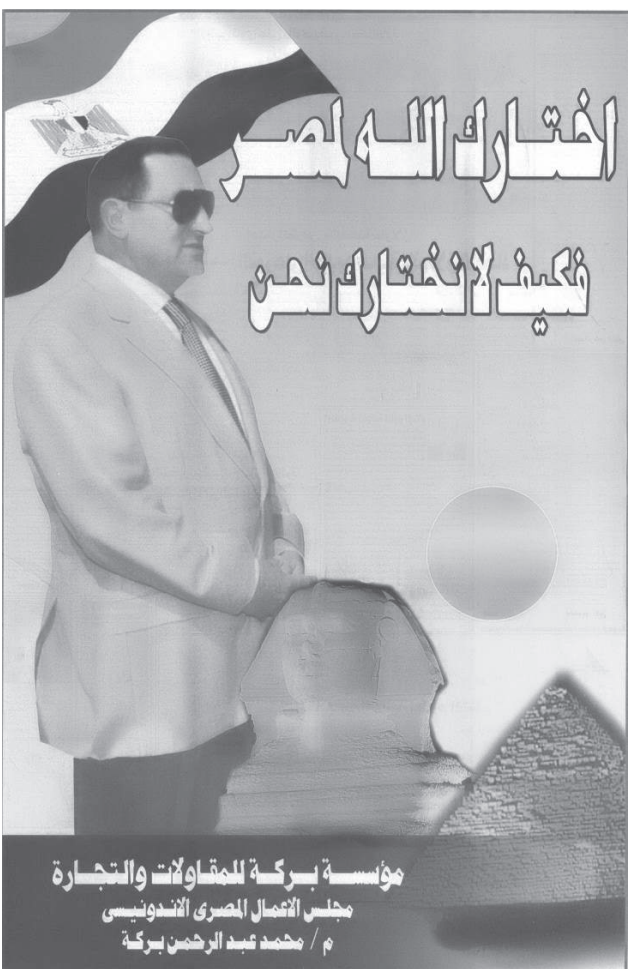

Document 3 : (Al-Akhbâr, 28/08/2005) côtés d'autres entrepreneurs du secteur privé opérant dans divers domaines d'activité et, de manière notoire, des PDG d'entreprise de construction et d'ingénierie civile qui s'accaparent les encarts les plus convoités.

En pleine campagne, ces publicités électorales venues du secteur privé sont l'occasion de messages très variés qui se rejoignent autour d'un même ton laudateur, voire obséquieux, à l'endroit de Hosni Moubarak, le président et/ou le candidat. Il peut s'agir de « renouveler l'allégeance au président M. H. Moubarak pour un nouveau mandat présidentiel afin de compléter le chemin

32. Luc Boltanski et Élizabeth Claverie, "Du monde social en tant que scène d'un procès ", art. cit., p. 419. 
de l'essor et de l'abondance » et de lui "souhait[er] toujours une bonne santé », à l'instar des directeurs de la sucrerie de Daqahliyya (Al-Akhbâr, 28/08/2005) ; ou plus "simplement », d'adresser un " merci et oui, de la part des fermes et des paysans du gouvernorat du Fayoum, pour le fils de l'Égypte protégée, son dirigeant, son leader (za'îm) et son aimé, M. H. Moubarak » (Al-Jumuhûriyya, 18/08/2005). L'annonceur peut également se faire le chantre des progrès du secteur d'activité le concernant. Ainsi, deux instituts privés d'enseignement supérieur dressent la liste des « succès de la période Moubarak dans l'enseignement supérieur (1981-2005) » (Al-Ahrâm, 2/09/2005) en regard de l'affiche officielle de la campagne du PND. Sur un photomontage personnel, le géant du secteur bâtiment et travaux publics 'Uthmân Ahmad 'Uthmân chante les louanges des développements du secteur de la construction publique « sous le sage commandement éclairé de son Excellence le président M. H. Moubarak, berger de la renaissance de l'Égypte moderne »(Al-Ahrâm, 1/09/2005).

Le directeur de l'entreprise ou le principal actionnaire signent généralement de leur nom, mais ils prennent la parole au nom de la population dont ils ont la direction ou qu'ils prétendent représenter. Le directeur d'un institut privé d'ingénierie civile s'exprime ainsi au nom des étudiants et des professeurs de son établissement dans les différents encarts qu'il publie ; trois directeurs de PME se posent en porte-parole des « masses de Kafr al-Shaykh disant oui à Moubarak» (Al-Jumuhûriyya 27/08/2005) ; le citoyen 'Amr Galâl Harîdî rend publique "la même intention des chefs, shaykhs et familles des villages du centre de Sâhil Salîm [...] qui [prêtent] allégeance et soutiennent Monsieur le président M. H. Moubarak» (cf. document 2). Des enjeux de nombre peuvent être mis en avant dans ce type de « représentation populaire » : le directeur de la société Al-Amîr pour la céramique et la porcelaine s'approprie de la sorte les voix de « 2800 Égyptiens travaillant à l'ombre d'un climat d'investissement et de sécurité » (Al-Ahrâm, 1/09/2005), de même que le P-DG Sulaymân 'Âmir s'exprime au nom des 2000 employés de la ville [touristique] al-Sulaymaniyya dont il est gérant.

Si certaines publicités concentrent leurs messages sur le texte, nombreuses sont celles qui reprennent I'affiche officielle de Moubarak et se contentent $d^{\prime} y$ ajouter une déclaration de soutien. D'autres encore proposent leurs propres créations en reprenant des photographies officielles de Moubarak et en puisant dans les symboles du patrimoine égyptien : temple de Louxor ou Citadelle de Salâh al-Dîn au Caire. L'annonce reproduite ci-dessus (Document 3) est publiée en quatrième de couverture $d^{\prime} A /$-Jumuhûriyya et est financée par le directeur d'une entreprise de travaux et de commerce. Le photomontage agence une photographie officielle de Moubarak avec celles de plusieurs symboles nationaux (le drapeau, le Sphinx et une pyramide du plateau de Gizeh), sous le slogan «Dieu t'a choisi pour l'Égypte, alors comment ne te choisirions-nous pas [?] ».

Ces publicités personnelles ne sont pas apparues avec l'adoption de l'élection comme mode de désignation de l'exécutif égyptien. On les retrouve dans 
la presse nationale à l'occasion des anniversaires de Moubarak et, en leur temps, lors des référendums. Si certains messages se situent dans une certaine continuité avec les messages qui fleurissaient à l'occasion des précédents référendums (" renouveler l'allégeance »; " oui à Moubarak »), d'autres adoptent une rhétorique appropriée au choix que suppose l'élection (" Pourquoi [voter] pour Moubarak ?»; " [Untel] soutient la candidature de Moubarak [plutôt que celle d'un autre] »). Certaines de ces annonces peuvent servir des objectifs spécifiques et propres à la compétition électorale, comme le décompte d'intentions de vote des employés d'une entreprise (cf. supra), l'appel au vote (" Donne ta voix au candidat du PND ») ou l'annonce des allocutions télévisées du candidat Moubarak (" Aujourd'hui, en direct sur les chaînes Dream : Son excellence le président $\mathrm{M}$. H. Moubarak présente son programme d'activation et d'opportunités de travail »).

L'une de ces campagne qui paraît sur un encart de une d'Al-Ahrâm pendant toute la durée de la campagne, mérite une mention particulière tant ses annonceurs, un groupe d'investisseurs anonymes de Sharm al-Shaykh, s'ingénient à étayer d'exemples la campagne électorale de Moubarak. En réutilisant le matériel de campagne de Moubarak - l'affiche, le logo " Moubarak 2005 » et le slogan de sa campagne ("le leadership et la passerelle vers le futur») ces investisseurs rendent public leur soutien (ta'yid) à Moubarak et mettent en regard les développements qu'a connus cette ville nouvelle avec les thèmes de campagne et les promesses électorales de Moubarak sous l'intitulé « Pourquoi [voter] Moubarak?». Quotidiennement, une thématique est mise en exergue, reprenant parfois mot pour mot les déclarations de Moubarak: "En seulement 15 ans, l'Égypte est devenue forte et sûre » (Al-Ahrâm, 4/09/2005) ; " En seulement 15 ans, ta vie est devenue meilleure » (Al-Ahrâm, 28/08/2005); "En seulement 15 ans, le citoyen s'est converti à la démocratie »(Al-Ahrâm, 27/08/2005). Ces thématiques sont ensuite mises en relation, respectivement, avec la tenue de conférences de paix à Sharm al-Shaykh, la construction de logements sociaux dans la station balnéaire ou, au niveau national, la création $d^{\prime}$ instances et de dispositions judiciaires en faveur des femmes. Les messages sont ponctués d'un slogan du choix des annonceurs : «Moubarak : des faits et des chiffres et pas seulement des slogans ».

Président sortant, Moubarak est le seul candidat à pouvoir déléguer, de la sorte et grâce à ces messages, le financement et la conception de tout ou partie de sa campagne électorale par voie de presse. À mieux considérer l'articulation entre publicités officielles et messages personnels, il semble que les conseillers électoraux du PND s'appuient largement sur cette délégation de la publicité électorale. Comme je l'ai souligné plus haut, les affiches officielles du candidat Moubarak ne paraissent qu'à deux reprises, en début et fin de campagne, ce qui semble indiquer que les messages publicitaires personnels de soutien à Moubarak font partie intégrante de la stratégie de campagne du PND. Cette délégation de campagne électorale par voie de presse revêt un double intérêt et permet à Moubarak de contourner deux 
dispositions règlementant les modalités pratiques de la campagne électorale. Le premier intérêt est d'ordre financier. Cette omniprésence publicitaire est supportée financièrement par des éléments extérieurs au Q-G de campagne, ce qui permet au candidat Moubarak de s'affranchir des règles inhérentes au financement de la campagne. Les dépenses engagées dans la campagne sont plafonnées à 10000000 LE alors que les frais d'une publicité nominative de soutien à Moubarak ne sont pas comptabilisés par la Commission de suivi des élections. Le deuxième intérêt concerne le temps règlementaire de la période électorale (deux semaines et demie) et la possibilité pour Moubarak de s'affranchir de cette contrainte grâce à la délégation de sa campagne électorale dans la presse nationale (sans compter les annonces privées passées dans la presse d'opposition). Les annonceurs privés n'étant pas tenus de respecter les dispositions de la loi électorale qui ne s'applique qu'aux seuls candidats, Moubarak bénéficie d'une longueur d'avance, dans ce domaine (comme dans tant d'autres) sur ses concurrents. Les premières publicités privées de soutien à Moubarak apparaissent ainsi dès le 13 août, soit cinq jours avant le début officiel de la campagne, et se poursuivent jusqu'à la publication des premiers résultats du scrutin, le 9 septembre, soit cinq jours après la date officielle de clôture de la campagne électorale. ${ }^{33}$

Sans mettre en doute la sincérité des sentiments qui animent ces annonceurs à l'égard du président Moubarak, on ne manquera pas de relever que ce type de publicité électorale ne sert pas uniquement les intérêts du candidat du PND. Ces messages publicitaires sont en effet le lieu d'une convergence d'intérêts entre le candidat Moubarak et les annonceurs privés. Dès l'annonce de la date officielle des élections présidentielle et législatives, nombreux sont les analystes de tous bords à articuler les deux rendez-vous de cet automne électoral. La proximité des élections législatives, un mois après la présidentielle, pèse de tout son poids dans les calculs des annonceurs privés. Au-delà des retombées commerciales généralement attendues d'une opération marketing, l'intérêt que ceux-ci trouvent dans la publication de publicités de soutien au président est de l'ordre de la cooptation, tant politique que commerciale. On a déjà souligné plus haut qu'un nombre non-négligeable d'annonceurs opéraient dans le secteur de la construction et de l'ingénierie civile. Ce sont autant de candidats aux appels d'offre de travaux publics que publie l'État. En matière de cooptation politique, les rétributions peuvent varier, mais le soutien du parti présidentiel pour un siège de député à l'Assemblée du peuple, est assurément la plus convoitée.

33. La campagne électorale prenait officiellement fin le 4 septembre, soit trois jours avant le scrutin. Cette concurrence déloyale du candidat Moubarak sur ses adversaires est aggravée par le fait que l'aide financière de 500000 LE octroyés par l'État aux candidats, et que seuls Moubarak et Gum'a ont décliné, a été versée plusieurs jours après le lancement officiel de la campagne électorale, retardant ainsi le début des activités de campagne des candidats anonymes. 
Le lien entre ce type d'annonce publicitaire et la cooptation politique et partisane est rendu manifeste par le recours à ce mode de communication politique par des députés en place. Là encore, le poids de la perspective des législatives reste entier : ces parlementaires-annonceurs cherchent à s'attirer le soutien du parti en vue d'une réélection ou d'une nomination à la tête d'une commission parlementaire. C'est surtout vers la fin de la campagne électorale et au moment du scrutin que l'on trouve des annonces signées par des parlementaires. Ceux-ci se démarquent des autres annonceurs - les hommes d'affaires alors extérieurs aux cénacles politiques - par le fait qu'ils se contentent rarement $d^{\prime}$ un simple soutien, considérant manifestement que, dans un espace publicitaire particulièrement concurrentiel, il faut se démarquer des autres annonceurs. Moins immédiatement concerné, un membre de l'Assemblée consultative, peut ainsi se satisfaire d'un petit encart en une et d'un simple message : "Oui à Moubarak. Remerciement, estime et soutien au zaî̀m M. H. Moubarak»(Al-Jumuhûriyya, 27/08/2005). Les députés de l'Assemblée du peuple, quant à eux, se doivent de rendre visible un soutien plus actif et une contribution plus concrète à la campagne électorale du candidat du PND, au-delà de la seule annonce publicitaire. Au milieu des pages d'actualité sportive, le député Muhammad Murshidî se targue d'avoir " conduit le plus grand rassemblement populaire à l'échelle de la République, à alMa'âdî, Tura et al-Basâtîn ; Plus de 20000 citoyens [y] ont assisté pour soutenir Moubarak» (Al-Ahrâm, 4/09/2005). La campagne électorale est l'occasion de créer l'événement et de prendre de l'avance sur ses futurs concurrents de circonscription. Candidat dans la circonscription d'al-Manyal en 2005 et en $2007^{34}$, Sayyid Salâma lance un « appel général à tous les citoyens d'Égypte, à ceux qui aiment I'histoire de la dévotion [à Moubarak] et ceux qui soutiennent M. H. Moubarak, candidat du PND » et organise " une conférence populaire au cinéma Fâtin Hamâma, pour que I'histoire de la dévotion continue » (Al-Ahrâm, 29/08/2005).

Peu avant la tenue du scrutin, alors que le taux de participation s'annonce faible, nombreux sont les parlementaires et/ou hommes d'affaires à lancer des appels au vote. La veille du scrutin, deux jours après la clôture officielle de la campagne, le P-DG Ahmad Khayrî de la société du même nom signe le message suivant sur une affiche de campagne de Moubarak: "Appel à voter : pour ce qui est à venir, selon la permission de Dieu l'Exalté, pour notre Égypte chérie : les masses du noble peuple égyptien, toutes confessions confondues, doivent aller aux urnes pour pratiquer leur droit électoral » (Al-Ahrâm, 6/09/2005). Les annonces accompagnent l'événement et les députés les plus fortunés s'offrent

34. En 2007, la députée du PND Sâfînâz al-Naggâr abandonne son siège après son mariage avec Ahmad 'Izz, I'homme d'affaire détenant le monopole de I'industrie sidérurgique en Égypte et homme d'influence de la direction du PND. L'élection est remporté par le candidat du PND Magdy 'Allâm le 24/12/2007. 


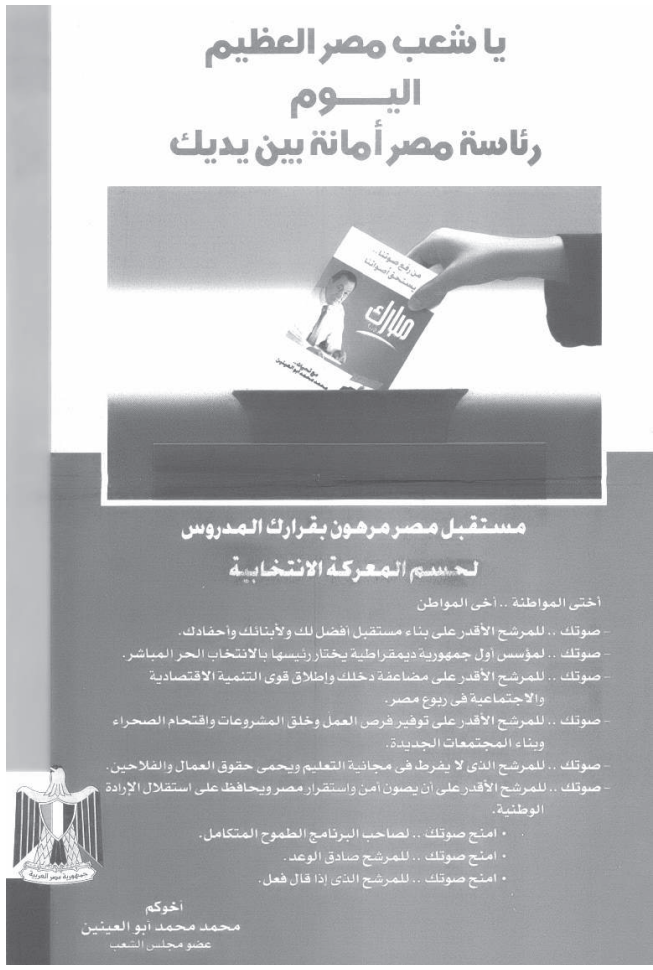

Document 4 (Al-Akhbâr, 28/08/2005)

une pleine page de quatrième de couverture pour appeler au vote. Le jour du scrutin, un homme d'affaires, député de la circonscription de al-Daqahliyya, lance un appel aux urnes avec des accents élogieux : «Aujourd'hui, le jour de la récolte et de la restitution des bienfaits, toute l'Égypte est avec toi " (Al-Akhbâr, 07/09/2005). Le même jour, le député de la circonscription de Gîzah, Muhammad Abû-I'Aynayn, également homme d'affaires, publie en quatrième de couverture d'Al-Ahrâm une affiche d'incitation au vote à la rhétorique particulière (cf. cicontre) : " Ô peuple suprême d'Égypte, aujourd'hui, la présidence d'une Égypte sûre est entre tes mains ». L'argument de la sécurité et de la stabilité fait écho à l'un des thèmes de campagne privilégiés de Moubarak dont on voit l'affiche de campagne en guise simplement qu'au terme des différentes compétitions électorales de 2005, AbûI-'Aynayn est reconduit à son poste de député et est nommé président de la commission parlementaires des Affaires étrangères.

\section{CONCLUSION}

Au terme de cet article, on a pu observer comment la presse nationale a apporté sa contribution à la campagne électorale et à la réélection du président Moubarak. L'examen de l'espace rédactionnel de ces journaux a montré comment certaines hiérarchies étaient cimentées par une politique éditoriale indéfectiblement loyale au " président Moubarak » et une neutralisation des discours d'opposition, parfois virulente, notamment avec le "challenger» Ayman Nûr. L'analyse de l'espace publicitaire de ces publications a révélé certaines subtilités de la publicité électorale et de la politique égyptienne et les jeux de clientèle avec les appels à l'aide, et de cooptation, avec les annonces de soutien. Cette analyse des deux parties complémentaires de la presse 
nationale a montré combien celle-ci constitue un lieu particulièrement fécond d'observation de l'ingénierie électorale et un prisme éminemment heuristique pour l'analyse de cette élection.

À l'issue du décompte des voix, Hosni Moubarak remporte 88,5\% des suffrages exprimés, devant Ayman Nûr (7,5\%), Nu'mân Gum‘a (2,9\%) et les sept autres candidats (moins de 1\%). Le 27 septembre, Moubarak est investi président de la République lors d'une cérémonie organisée en grandes pompes à I'Assemblée du peuple. En bon maître de cérémonie, le président du Parlement, Fathî Surûr, introduit le victorieux Moubarak devant les députés, sous l'œil attentif des hauts-gradés de l'armée, des grands noms du régime et d'un parterre d'invités au nombre desquels le colonel al-Qadhâfî. Le lendemain de l'investiture, le cérémonial protocolaire est fidèlement restitué par Al-Ahrâm qui consacre les trois premières pages de sa maquette, les deux hauts-lieux de l'événementiel dans les rubriques du journal, à la couverture de la cérémonie illustrée de larges photos en couleur. Et la rédaction de titrer en manchette : "L'ère du président élu a commencé » (28/09/2005). Derrière la solennité du cérémonial, on assiste à la reformulation des bases de légitimité sur lesquelles le président peut désormais s'appuyer et fonder son action. Le président Moubarak issu du référendum fait place au président Moubarak élu. La continuité, dont l'indice le plus tangible est la reconduction de Moubarak à son poste, se fond ici dans le changement ainsi performé par la vertu de l'élection et du suffrage universel. 